\title{
Étude d'une nouvelle source optique UV-VUV basée sur une décharge multicanaux de forte puissance
}

\author{
V.I. Tcheremiskine ${ }^{1,2}$, M.L. Sentis ${ }^{1}$, L.D. Mikheev ${ }^{2}$ et M. Sabonnadière \\ ${ }^{1}$ Laboratoire Lasers, Plasmas et Procédés Photoniques (LP3), FRE 2165 du CNRS, Université \\ d'Aix Marseille II, Campus de Luminy, Case 917, 13288 Marseille cedex 9, France \\ ${ }^{2}$ Laboratoire des Processus Photochimiques, Institut de Physique P.N. Lebedev, Leninsky \\ Prospekt 53, 117924 Moscou, Russie
}

Résumé : Une nouvelle source plasma VUV de grande dimension $\left(20 \times 50 \mathrm{~cm}^{2}\right)$ et de forte température de brillance $(\mathrm{T}>30000 \mathrm{~K})$ dans le domaine du VUV est présentée. Cette source constitue le moyen de pompage d'un amplificateur pour laser femtoseconde dans le domaine du visible.

\section{INTRODUCTION}

Le développement de sources incohérentes de puissance émettant dans les domaines de l'UV et du VUV est important pour différentes applications scientifiques et industrielles, telles que le pompage de laser, le traitement des eaux et des gaz, la désinfection et la décontamination de divers objets, etc.

Une telle source plasma de grande dimension est généralement produite par une décharge rampante le long de la surface d'un diélectrique initiée par une décharge de barrière diffuse [1]. Cependant, cette source, généralement appelée "décharge de plasma plane", se caractérise par une densité du courant électrique relativement faible. Cet inconvénient conjugué à la diminution du coefficient d'absorption du plasma lorsque la longueur d'onde diminue, fait que la brillance de ce type de décharges atteint difficilement une intense luminosité dans le domaine spectral de l'UV et en particulier du VUV. Une autre méthode prometteuse est le déclenchement d'une décharge rampante se composant de nombreux canaux de plasma de faible diamètre tel est l'objet du présent travail.

\section{DESCRIPTION DU DISPOSITIF EXPERIMENTAL}

Le principe de déclenchement d'une décharge multicanaux ainsi que des résultats préliminaires concernant les mécanismes du développement de ces décharges ont déjà été présentés [2a]. Dans le présent travail, des études de caractérisation électrique, énergique et spectrale d'une nouvelle source optique UV et VUV constituée de 47 canaux de décharge sont présentées. Afin d'augmenter la puissance de rayonnement, un circuit électrique rapide a été conçu; il fonctionne sans commutateur de puissance. Les 47 canaux de décharge fonctionnent en parallèle (Fig. 1). Ils ont une longueur de $20 \mathrm{~cm}$ et sont distants les uns des autres de $1,2 \mathrm{~cm}$. Ils sont alimentés par un banc de condensateurs d'une valeur de 4,8 $\mu \mathrm{F}$. Les décharges sont effectuées dans un mélange $\operatorname{Ar} / \mathrm{N}_{2} 2 / 1$ à la pression totale de $1 \mathrm{~atm}$.

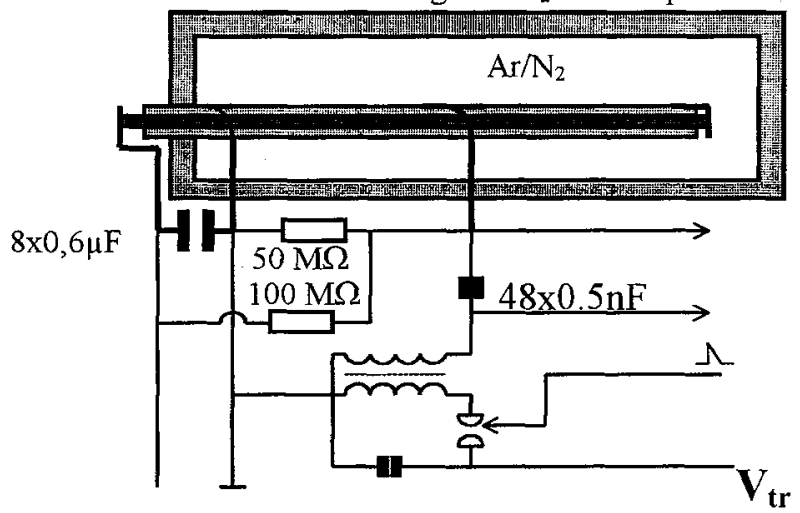

Fig. 1 : Représentation schématique du dispositif expérimental 


\section{RESULTATS}

Le circuit électrique réalisé permet un accord d'impédance (plasma-circuit) remarquable et un dépôt d'énergie de $4 \mathrm{~kJ}$ dans un intervalle de temps de seulement $1,5 \mu \mathrm{s}$. On observe à l'aide d'une caméra CCD (résolution temporelle de 10 ns) une excellente homogénéité spatiale et une parfaite synchronisation des 47 plasmas de décharge comme le montre la figure 2 .

vers les autres

décharges

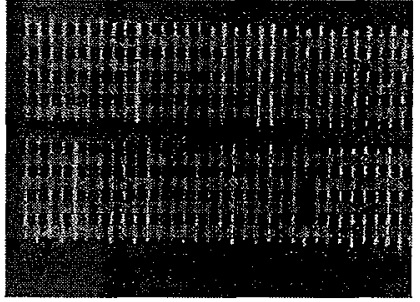

(a)

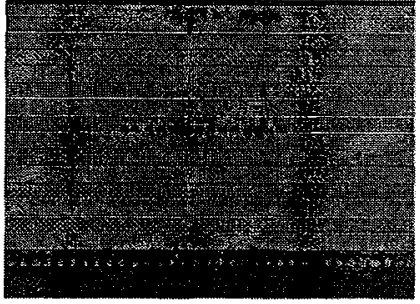

(b)

Fig. 2 : Photographies des plasmas de décharges ; a) déclenchement, b) décharge principale

Les spectres observés (Fig. 3) montrent qu'environ la moitié du rayonnement émis dans le VUV est associée aux transitions d'atomes et d'ions d'argon et azote. L'autre moitié est due au continuum d'émission spectrale. La vitesse d'expansion observée d'un canal plasma est d'environ $2 \mathrm{~km} / \mathrm{s}$. À l'instant où le rayonnement UV-VUV atteint son maximum $(t \approx 0,9 \mu \mathrm{s})$, la surface de rayonnement de la source excède alors $1000 \mathrm{~cm}^{2}$. Les études expérimentales par actinométrie dynamique montrent que la température de brillance du plasma ainsi produite est alors de $30 \mathrm{kK}$.

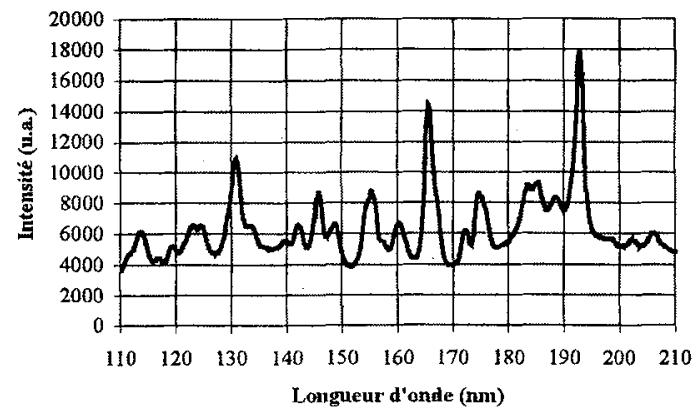

Fig. 3 : Spectre d'émission de la source VUV pour un mélange $\mathrm{Ar} / \mathrm{N}_{2}$ de 1 bar.

\section{CONCLUSION}

Les caractéristiques de cette source ainsi réalisée permettent la conception d'un pompage homogène par photoexcitation d'un milieu laser placé entre deux sources optiques[ $2 b$ ]. Cette source servira à étudier les mécanismes d'excitation de lasers photochimiques et la possibilité d'amplification directe d'impulsion laser femtoseconde jusqu'à une puissance de l'ordre du petawatt dans le domaine spectral du visible.

\section{Remerciements}

Les auteurs remercient INTAS pour son support à travers le programme : 97-1869.

\section{Références}

1. R.E.Beverly III, J. Appl. Phys. 60, 104-124 (1986).

2. V.I.Tcheremiskine, M.L.Sentis, et al., "Proceedings of the XXIII International Conference on the Phenomena in Ionized Gases" (Toulouse, 1999), a) Vol. IV, 52; b) Vol. I, 48. 